\title{
Cancer Stem Cells in the Personalized Therapy of Colorectal Cancer
}

\author{
Athanasios Patsalias, Zuzana Kozovska* \\ ${ }^{1}$ Laboratory of Molecular Oncology, Cancer Research Institute, Biomedical Research Center of SAS, Dubravska \\ cesta 9, 84505 Bratislava, Slovakia; athanpatsalias@gmail.com \\ * Correspondence: zuzana.kozovska@savba.sk; Tel.: +421-232 295138
}

Abstract:

BACKGROUND: Treatment failure in primary as well as metastatic cancer patients, caused by chemo and radio resistance, has truncated the research for the applicability of personalized medicine. The use of stem cells and cancer stem cells in such a treatment approach will be reviewed in this study.

RESULTS: CRC stem cells prove to be a promising asset for CRC treatment optimization both by serving as biomarkers for the current therapy modalities by means of treatment personalization and patient/tumor stratification, as well as in the development of targeted therapies, selective for the stem cell population. Similar conclusions are drawn, regarding mesenchymal stromal cells and their effect in CRC therapy; while resident stromal cells of tumor microenvironment seem to promote the tumorigenic and metastatic processes in addition to conferring to the chemo- and radio resistance, under certain conditions they are able to improve the treatment outcome of CRC chemotherapy, e.g. by targeted enzyme/prodrug treatment of CRC cells.

CONCLUSION: This review, truncates the dynamic potential of cancer stem cells and other stem cell types in CRC treatment personalization as well as, in the improvement of current treatment approaches opting to a higher therapeutic rate, improved prognosis, survival and quality of life for CRC patients.

Keywords: colorectal cancer, cancer stem cells, mesenchymal stromal cells, stem cell markers, chemoresistance, treatment personalization, biomarkers, cancer stem cell markers.

\section{Introduction}

Cancer is a group of diseases, characterized uncontrolled cellular proliferation, tissue invasion and metastasis. Since it constitutes one of the major factors of fatality in our era, scientific research has successfully developed a very wide armory of antineoplastic drugs with a variety of pharmacological properties, including the novel forms of targeted anticancer therapy. Cancer, however, is enormously complex and heterogeneous and the current gaps in our knowledge preclude the vision of total success in its therapy from becoming a reality in the very near future, with phenomena such as chemo- or radiotherapy failure and subsequent tumor relapse and metastasis, as well as inter-individual variabilities towards anticancer treatment efficacy, decreasing the overall survival rates and indicating the need of new, personalized and targeted anticancer treatment modalities, which shall "dodge the bullets" of treatment failure as well as treatment response variability among patients of different or, frequently the same types of neoplasia. The "vision" of personalized medicine, involves measurement of some "key" tumor characteristics, which assist the optimum therapy regimen being tailored for each patient to successfully treat their cancer and prevent relapse. Recent years have seen researchers and clinicians alike take great strides toward personalized cancer care. The advent and use of new high-throughput technologies has resulted in a data explosion in the field of cancer research; the challenge over the coming years will be to bridge the gap between the bench and bedside. In this article, we opt to review systematically, the most recent advances in the field of personalized medicine towards a very common and unfortunately, lethal disease, namely colorectal cancer with a focus on the most up to date evidence in the role of stem cells and cancer stem cells, in this field. 


\subsection{Colorectal Cancer: overview of the disease}

\subsubsection{Statistics}

Colorectal cancer (CRC) is the third leading cause of cancer death worldwide, and the 5-year relative survival rate is only $8 \%$ despite diagnostic and therapeutic advances [1]. Despite the existence of excellent screening and preventive strategies, (CRC) remains a major public health problem in Western countries. About $72 \%$ of new CRCs arise in the colon, and the remaining $28 \%$ arise in the rectum [2].

\subsubsection{Histopathology and genetics of CRC}

The main histopathological classification of CRC includes: Adenocarcinomas (90-95\% of total large-bowel neoplasms), malignant tumors derived from cuboidal or columnar epithelial cells; Mucinous adenocarcinoma, a histologic variant characterized by huge amounts of extracellular mucus in the tumor and the tendency to spread within the peritoneum (approximately $10 \%$ of colorectal adenocarcinomas are mucinous, more commonly seen in younger patients); Signet-ring cell carcinoma is an uncommon variant, accounting for $1 \%$ of colorectal adenocarcinomas; other tumor types, include: Squamous cell carcinomas, small-cell carcinomas, carcinoid tumors, and adenosquamous and undifferentiated carcinomas also have been found in the colon and rectum. Nonepithelial tumors, such as sarcomas and lymphomas, are exceedingly rare [2].

Tumorigenesis occurs when cells acquire six hallmarks: self-sufficiency in growth signaling, insensitivity to anti-growth signaling, evasion of apoptosis, unlimited replicative potential, sustained angiogenesis and tissue invasion. An initial event of cancer involves genetic defects that cause DNA instability activation followed by less tumor suppressor gene or gatekeeper pathway [3].

Screening of CRC cells with DNA probes specific for known protooncogenes and some tumor suppressor genes revealed that $50 \%$ of these malignant tumors have acquired an activating point mutation of K-Ras oncogene and $75 \%$ of these cancers had an inactivating mutation on the $p 53$ tumor suppressor gene. Moreover, studies of multiple cases of Familial adenomatous polyposis (FAP), have shown that more than $70 \%$ of colon cancers derived from intestinal polyps have a mutation in the Adenomatous polyposis coli (APC) gene. Indeed, it now seems clear that mutations inactivating the APC gene, represent an early stage [4].

Progression of colon normal epithelium to invasive carcinoma goes through several stages. The invasive carcinoma stage involves epithelial cells losing their polarity and detaching from the basement membrane altering cell-ECM interactions and signaling networks producing changes in stem cells that generate cancer stem cells. Malignant phase of tumor growth can progress from this stage to metastatic cancer, also involving invasion of tissue by feed blood vascular systems [3].

\subsection{Current CRC treatment options overview}

CRC treatment is considered to be either local therapy or systemic therapy. Local therapies consist of surgery, radiation therapy and interventional radiology. Systemic therapy consists of chemotherapy and immunotherapy, for these drugs enter the bloodstream and destroy or control the neoplastic cells throughout the body [5].

Depending on the stage of the cancer and other factors (age, pathophysiological status, gender, types of mutations, etc.), different types of treatment may be preferred, combined at the same time or used after one another: early stages of CRC ( $\sim 95 \%$ of stage I and $\sim 70 \%$ of stage II) commonly receive surgery, with or without adjuvant chemotherapy; Advanced stages (III and IV), commonly receive combination therapies, including chemotherapy radiation and surgery [6].

\subsubsection{CRC chemotherapy}

In CRC, chemotherapy is usually given after surgery of stage III tumors, where the carcinoma cells have spread to lymph nodes (adjuvant chemotherapy). In this way, chemotherapy may help reduce the risk of cancer recurrence and death from cancer. Sometimes chemotherapy may be used before surgery as well, with the goal of shrinking the cancer before an operation [7]. Chemotherapy before surgery is more common in rectal cancer than in colon cancer. Chemotherapy can also be given 
to relieve symptoms of colon cancer that has spread to other areas of the body (palliative chemotherapy). Finally, patients presenting with advanced (unresectable) metastatic CRC (mCRC) receive chemotherapy as a treatment of choice, which has highly improved the prognosis and increased the median survival rate of such patients [8].

First line chemotherapeutic agent 5-fluorouracil (5-FU), is the mainstay chemotherapeutic agent in most CRC cases [5]. The development of additional agents, however, including platinumcontaining agents, irinotecan, oral fluoropyrimidines and raltitrexed has expanded the chemotherapeutic "arsenal" for CRC patients. The choice of combination chemotherapy is available also, mainly for stage IV (advanced) metastatic CRC, with a profound synergistic toxicity advantage; the same benefit is nowadays evident for combinations in adjuvant (post-resection) chemotherapy of CRC $[9,10]$.

\subsubsection{Current CRC immunotherapy overview}

The limitations of surgery and adjuvant chemo/radio/antibody therapies to treat CRC patients necessitate the development of novel approaches, including immunotherapy [11]. Immunotherapy is an active therapeutic approach designed to trigger the immune system to respond to tumor-specific antigens and attack tumor cells. Immunotherapy strategies include the use of peptides derived from tumor-associated antigens, whole tumor cells, in vitro-generated dendritic cells, or viral vector-based cancer vaccines [12].

Moreover, monoclonal antibodies (mAbs) that target surface antigens expressed on tumor cells are clinically effective as cancer therapeutics [13]. Three mAbs (Cetuximab, Bevacizumab and Panitumumab) are approved for the treatment of CRC in the United States, and many other mAbs are being tested in clinical trials. Bevacizumab, a recombinant humanized monoclonal antibody that selectively binds to human VEGF, is effective in KRAS wild-type CRC patients [14]. Recent evidence has also shown clinical benefits from treatment with anti-EGFR, Cetuximab and Panitumumab in KRAS wild-type CRC patients [15].

\subsection{Resistance of CRC to current treatment options}

One of the primary causes for therapeutic failure in cancer is resistance to radiation and chemotherapeutic drugs. Tumors usually consist of a mixed population of malignant cells, some drug-sensitive, and some drug-resistant. Cancer cells that show resistance to chemotherapy when first exposed to an anticancer drug have intrinsic MDR. Chemotherapy usually destroys drugsensitive cells, and favors the survival of drug-resistant cells. More tumors, however, may initially be sensitive to therapy and later become insensitive to similar drugs, having acquired resistance [16]. After an initial treatment with chemotherapy, the tumor begins to grow again and develop resistance to chemotherapeutic agents. Cancer cells employ several mechanisms to resist chemotherapy and radiotherapy (Table 1) [17].

When irradiation is unable to reduce tumor volume or cancer recurs after a regression, then a cell is considered to be radioresistant. The curative potential of radiotherapy is limited by intrinsic radioresistance of cancer cells. The degree of radiosensitivity is recognized by both intrinsic and extrinsic properties [18].

Table 1 Mechanisms of chemo- and radio resistance of cancer cells

\begin{tabular}{|c|c|}
\hline Radioresistance & Chemoresistance \\
\hline \hline Tumor hypoxic conditions & Increased drug efflux \\
\hline Increased production of cellular antioxidants & Decreased or limited drug uptake \\
\hline \hline Activation of certain protooncogenes & Evasion/Inactivation of apoptosis \\
\hline Stromal interactions & $\begin{array}{c}\text { Increased Drug metabolism and drug } \\
\text { compartmentalization }\end{array}$ \\
\hline
\end{tabular}




\begin{tabular}{|c||c||}
\hline Amplification of DNA repair (caretaker) genes & Increased efficiency of DNA repair mechanisms \\
\hline \hline $\begin{array}{c}\text { Use of epigenetic mechanisms that promote cell } \\
\text { survival }\end{array}$ & $\begin{array}{c}\text { Increased expression or expression of altered drug } \\
\text { targets (e.g. enzymes, structural proteins, etc.) }\end{array}$ \\
\hline \hline Alterations in "checkpoints" of cell cycle & Survival signals favored by transcription factors \\
\hline \hline
\end{tabular}

\section{The emerging role of stem cells in CRC treatment}

Advances in understanding adult stem cell biology have facilitated the development of novel cell-based therapies for cancer. Recent developments in conventional therapies (eg, tumor resection techniques, chemotherapy strategies, and radiation therapy) for treating both metastatic and primary tumors in the brain, particularly glioblastoma have not resulted in a marked increase in patient survival. Preclinical studies have shown that multiple stem cell types exhibit inherent tropism and migrate to the sites of malignancy. Recent studies have validated the feasibility potential of using engineered stem cells as therapeutic agents to target and eliminate malignant tumor cells in the brain.

\subsection{The effect of mesenchymal stromal cells on human CRC cells}

The tumor microenvironment (TME) is an important factor in determining the growth and metastasis of colorectal cancer; the TME is composed of many cell types including tumor, stromal, endothelial and immune cell populations. It is widely accepted that cells present in the TME acquire distinct functional phenotypes that promote tumorigenesis. One such cell type is the mesenchymal stromal cell (MSC). Evidence suggests that MSCs exert effects in the colorectal TME, including the promotion of angiogenesis, invasion and metastasis [19]

Although the vast majority of the evidence points to a tumor promoting role for MSCs, there is some evidence to the contrary. However, this anti-tumor effect appears to be specific to the very early stages of tumor development. Throughout the course of a chronic inflammatory condition like inflammatory bowel disease (IBD) the epithelium becomes inflamed and damaged, leading to the production of factors such as nuclear factor kappa B (NF-kB), signal transducer and activator of transcription (STAT) 3 and STAT6, all of which are potentially tumorigenic [20]. It appears that administration of MSCs at this very early stage can have a tumor inhibiting effect by decreasing interleukin (IL)-6 and phosphoSTAT3 signaling and reducing DNA damage. However, once this early stage has passed, MSCs recruited to the tumor by factors such as nuclear factor (NF)- $k B$, chemokine receptor type 4 (CXCR4), stromal cell derived factor (SDF)1, monocyte chemotactic protein (MCP)-1 and vascular cell adhesion molecule (VCAM)-1 serve only to promote tumorigenesis via the mechanisms mentioned throughout this review, namely differentiation to cancer-associated fibroblasts $(\mathrm{CAFs})$, promotion of tumor growth, invasion, metastasis and angiogenesis and the dampening of anti-tumor immunity [20-22].

\subsection{The effect of MSCs in chemo- and radioresistance of CRC cells}

A final important aspect of CRC-MSC interactions is the potential impact that these cells could have on colorectal cancer therapy. Recent research has highlighted the ability of MSCs to contribute to resistance to chemotherapeutics in both hematological malignancies and solid tumors. In CRC, 5Fluorouracil (5-FU) is one of the first-line therapies for metastatic disease. However, it has now been shown that a population of bone-marrow MSCs exist that are both potently immunosuppressive and 5-FU resistant, a dangerous combination in the context of CRC chemotherapy. Difficulties also arise with the use of radiotherapy. MSCs have been shown to be radio-resistant and so could withstand such therapy and retain their tumor-conditioned phenotype, thus potentially contributing to disease relapse. Because of this reason there were constructed genetically modified MSC which are used in the enzyme/prodrug therapy. This therapy can be targeted selectively against the cancer cells. This 
cells then continuously express a transgene for the enzyme converting non-toxic compound (prodrug) into cytotoxic drug (suicide gene). There are several used enzyme/prodrug combinations:

1. herpes simplex virus-thymidine kinase with prodrug ganciclovir (HSVtk/GCV) [23,24],

2. yeast or bacterial cytosine deaminase (CD) alone or fused with uracil phosphoribosyltransferase (CD::UPRT) converting 5-fluorocytosine (5FC) to toxic 5fluorouracil (5FU) [25]

3. prokaryotic purine nucleotide phosphorylase in combination with fludarabine (PNP/Fara) [26]

Genetically modified MSC were successfully used in the treatment of colon cancer [27]

\subsection{Cancer stem cells and CRC}

Experimental evidence suggests, that a small sub-population of tumor cells, termed cancer stem cells (CSCs), are responsible for propagating cancer in a highly efficient manner. This malignant clonal population constitutes $0.1-10 \%$ of all tumor cells of which only some have the ability to form tumors [3]. Compared to normal stem cells (SCs), CSCs are thought to show no restraint with respect to cell number (i.e., proliferation); however, their slow rate of cycling plays a role in resistance to treatment (chemotherapy and radiotherapy) and tumor recurrence. Also, the ability of CSCs to initiate new tumors may be of critical importance for metastatic colonization [28].

In the CRC cancer stem cell model, malignant changes are suggested to start at the stem or precursor cell level. Precursor cells are a type of partially differentiated stem cell which has the capacity to differentiate into one cell type, and therefore are also called unipotent stem cells. Epigenetic changes, such as aberrant methylation, may result in silencing of genes p16, SFRPs, GATA-4/-5 and APC in stem/precursor cells of adult cell renewal systems and may lock these cells into stem-like states that foster abnormal cell clonal expansion, and the stem/precursor cells are transformed into pre-invasive cancer stem cells. At this stage, pre-invasive cancer stem cells turn into cancer stem cells that will ultimately become cancer cells due to accumulation of epigenetic and genetic abnormalities [29]

Several studies have suggested that the CSC fraction may be identified within a variety of human cancers, including CRC. Human colorectal CSCs were first isolated on the basis of CD133 expression and demonstrated to induce tumors in mice that resembled the original malignancy [30,31]. The search for other surface markers of colorectal CSCs proceeded in hopes of finding a CSC-specific biomarker, which would greatly facilitate the development of prognostic and therapeutic tools. Several CSC phenotypes have been described. However, CSC surface markers identified so far are expressed also by normal SCs, preventing their potential use as therapeutic targets. Several molecules have been proposed as colorectal CSC markers, including CD133, CD44, CD24, CD166, Lgr-5, and aldehyde dehydrogenase 1 (ALDH1). CD133, a pentaspan transmembrane glycoprotein, was one of the first colorectal CSC markers to be identified [32].

The cell adhesion molecule CD44 has been identified as a cell surface marker associated with CSCs in several types of tumor. CD44+ cells exhibited CSC properties, and a single cell could form a sphere in vitro, and a xenograft tumor resembling the original lesion in vitro. Overexpression of CD44 in CRC has been associated with depth of invasion and lymph node involvement and is shown to be an independent predictor of overall survival [33].

In addition to cell surface markers, activities of certain pathways or enzymes may also act as markers of stemness. For instance, normal colorectal stem cells can be identified by the activity of ALDH1, a detoxifying enzyme that oxidizes intracellular aldehydes [34].

Other markers include CD166, epithelial cell adhesion molecule, CD29, CD24, CD26, Msi-1, Lgr5 , and Wnt activity/ $\beta$-catenin. The presence of these molecules has been associated with stemness characteristics both in vitro and in vivo. These markers were also used to enrich isolated CSCs further to enhance their tumorigenic ability [35]. The transcription factors Oct-4 and Sox 2 are also promising CSC markers, given their roles in cell renewal. Oct-4 and Sox 2 levels have been shown to be elevated in CRC and to correlate with increased CSC proliferation and poor prognosis [36,37]. Other 
pluripotency genes, such as, Nanog and $c-m y c$, are regarded as promising surrogate markers, given that they appear to facilitate a shift towards an "undifferentiated" state. [38].

\section{Colorectal cancer stem cells and CRC - treatment personalization}

The proposal of the CRC stem cell (CRC-SC) model has great significance in guiding CRC therapy. The current chemotherapies generally aim at mature cancer cells, not the CRC-SCs. Although these treatments can reduce the size of cancer tissue, they cannot completely kill CSCs that have higher proliferative potential and stronger resistance to chemotherapy than mature cancer cell; they can "escape" the chemotherapy and differentiate into mature cancer cells when treatment is withdrawn, resulting in cancer recurrence and metastasis. Therefore, development of therapy targeting colorectal cancer stem cells, has a therapeutic potential to achieve better treatment to suppress cancer growth and metastasis.

\subsection{The role of CRC stem cells in CRC chemotherapy and chemoresistance}

Two models, have been proposed to explain the origin of CSC multidrug resistance (MDR) in tumors with an elevated ability to survive conventional chemotherapeutic regimens. The first model proposes that after exposure to the chemotherapeutic agent, only the CSCs expressing $A B C$ transporters are able to repopulate the tumor by asymmetrical cell division with newly-formed CSCs and/or differentiated progenitor cells. The second acquired model suggests that after chemotherapy, only CSCs survive and, those that acquire drug resistance under the pressure of mutations, originate new and more aggressive drug-resistant cell phenotypes [39].

Traditional cancer therapies, including chemotherapy and radiotherapy, depend on the rapid cycling cell cycle and aim at specific cell division phase, such as 5-fluoroucil (5-FU), the inhibitor of thymidylate synthase in cell mitotic S-stage, and oxaliplatin, a platinum compounds agent [40]. Nevertheless, there is evidence that intrinsic chemoresistance in colorectal cancer stem cells (CRC $\mathrm{SCs})$, may be associated with the slow growth of CRC-SCs in G0 stage. A large sample of clinical research conducted in 501 human CRC patients demonstrated that, tumor cells with CD133 overexpression showed increased resistance to 5-FU based chemotherapy and the expression of CD133 related to poor prognosis and decreased survival [41]. In oxaliplatin treated SW620 and LoVo CRC cells, overexpression of CD133 was also observed [42].

Recently, it was found that the high concentration of 5-FU and/or oxaliplatin treated human HT29 CRC cells, showed enrichment of CD133 + and CD44 + CRC-SCs and decreased in vitro proliferation [43]. Moreover, Insulin-like growth factor receptor I (IGF-1R), is found to be overexpressed in chemoresistant HT-29 cell line, while treatment with IGF-1R inhibitor, AVE-1642, can inhibit the growth of in vivo xenograft model. Other studies reported that human CD133 + CRCSCs can regulate death receptors and inhibit chemotherapy-induced cell apoptosis through specifically expressed interleukin-4 (IL-4). Treatment of CD133 + CRC-SCs, with anti-IL-4 antibody can increase the effectiveness of 5-FU or oxaliplatin-based therapy [44].

A recent analysis, reported an additional mechanism of CD133 + CRC-SCs 5-FU and oxaliplatin resistance: Aurora-A kinase, a regulator of mitosis affecting the process of cell cycle, is overexpressed in CRC-SCs; Aurora-A silencing can result in growth inhibition, down-regulation of anti-apoptotic protein expression and increase of chemotherapy sensitivity, which then, additively induce the death of CRC-SCs [45].

MicroRNAs (miRNA) also play a role in the regulation of cell proliferation and can regulate CRCSCs specific signal pathway to increase CR-CSCs drug-resistance. For example, miRNA-140 inhibits the proliferation of CD133 thigh /CD44 thigh human CRC cells through regulating histone deacetylase 4, leading to resistance to methotrexate (MTX) and 5-FU [46].

In the treatment of CRC and more importantly, metastatic CRC, chemotherapy effectiveness is also negatively impacted in regimes which include several chemotherapeutic anticancer agents of various pharmacologic groups (having different and diverse mechanisms of anticancer action), with this phenomenon being addressed as multi-drug-resistance (MDR). Reduction of the chemotherapeutic agent(s) concentration in the tumor cells, is the most studied mechanism of MDR: 
it is found that part of the tumor cells express adenosine triphosphate (ATP) depended on drug efflux transporter p-glycoprotein (P-gp, MDR1, ABCB1). Furthermore, in melanoma cells, ABCB5 resisted to camptothecin and 5-FU, which are two commonly used anticancer drugs, also in the case of CRC [47].

CSC surface markers identified so far are expressed also by normal SCs, preventing their potential use as therapeutic targets. In contrast to these surface marker molecules, the ALDH as a CRC-SC marker represents an intracellular protein with an enzymatic function executing the oxidation of both endogenously and exogenously produced aldehydes to their respective carboxylic acids. High activity of aldehyde dehydrogenase interfere with several chemotherapeutics used in the treatment of patients with colorectal cancer. Detoxification and drug inactivation represents one of the mechanisms contributing to chemoresistance. Recent data provides the evidence for CRC-type specific ALDH isoform expression: by silencing of ALDH1A1 or ALDH1A3 in tested cells we were compared chemosensitivity of these cells to unaffected ones. Samples after silencing were exposed to 5-FU, Capecitabine, Raltitrexed and Irinotecan, commonly deployed anticancer agents for the treatment of primary, as well as metastatic CRC. Silencing of these two ALDH1A isoforms led to different effect on chemosensitivity to tested drugs [48].

Finally, the effect of pharmacological inhibition of several ALDH isozyme activities by the chemical diethylaminobenzaldehyde (DEAB) partially sensitized the tested cell lines to chemotherapeutics: Chemosensitivity test in the presence of a sub-inhibitory concentration of DEAB, confirmed its potential to increase the antiproliferative effect of 5-FU, Irinotecan, Raltitrexed and Cisplatin in colorectal carcinoma cells [48].

\subsection{The role of CRC stem cells, as biomarkers for CRC personalized medicine}

Currently, most of the clinical trials are carried out on metastatic cancer patients with the aim of temporarily controlling tumor progression. However, with the increasing knowledge of colorectal CSC (CR-CSC) biology, several therapeutic options are becoming available. Current therapies do not target the CSC; this could be the reason behind recurrence of tumor growth or resistance to treatment post therapy [29].

CSCs have been isolated from leukemia, breast, prostate, brain, pancreatic, colon, and other types of cancer using either cell surface marker-based (e.g., CD44+, CD24, CD133+, etc.) or dye-effluxbased side population identification combined with cell sorting [49]. The prognostic value of CSC markers in the clinic is expanding, since it has been shown that CSCs are markers for poor patient prognosis [50,51]. Studies on CSC markers for colorectal cancer have shown that tumors harboring higher levels of both CD44 and CD133 had a higher risk of developing early liver metastases [52].

CSC markers are not only important for their identification but can also prove to be useful treatment targets [53]. Moreover, such markers may assist in proper selection of regimen of therapy and current status of treatment as well as disease: the primary aim of a predictive biomarker is to accurately determine the outcome of a given treatment. Ideally both, tumor and surrounding normal tissue responses can be assessed. If a biomarker is to be measured during the course of treatment, the accurate prediction should be made early enough to permit treatment interventions. This underlines the causative relationship between a biological factor and therapy outcome, i.e. measures to change biomarker expression lead to the corresponding change in treatment response. In other words, a strong association between biomarker and treatment outcome should result from a similar direct treatment effect on both, biomarker level and endpoint of therapy [54].

The aim of a biomarker - based patient stratification, for future treatment individualization trials, must be to identify patient groups whose chance of tumor cure after current standard treatment is approaching $100 \%$ and/or patient groups with poor tumor control rates. Such patient groups can then be brought into treatment de-escalation or treatment intensification trials, respectively. In contrast, for patients between these extremes it is currently extremely difficult to design a personalized interventional strategy which can be tested in clinical trials without major risk to reduce their individual chance of uncomplicated tumor control. For these patients, traditional clinical trials, accompanied by translational studies into refined biomarker panels, appear currently as the best 
strategy. The definition of extremely good or very poor prognosis group very likely requires the application of several biomarkers, where CSC markers are only one of them [55].

Some tissue-specific and whole genome approaches for molecular profiling of complex gene signatures of tumors and cancer cell lines have been developed in the last few years and led to the discovery of specific gene sets related to particular cancer types and which may predict the risk of metastatic dissemination at specific tissues and organs and overall outcome of patients with cancer. Moreover, the in vitro and in vivo characterization of stem cell markers and functional features of cancer stem/progenitor cells in tumor tissue specimens from patients with cancer and cancer cell lines has also revealed their major implications in tumor growth, metastases, treatment resistance, and disease relapse $[17,56]$.

In a recent study, drug responses to cisplatin and ALDH targeting compound or JAK2 inhibitor determined if the ovarian CSC population could be targeted. Cells that escaped therapy were isolated and used to initiate new spheroids and model tumor re-emergence in a personalized manner. The study concluded that, ovarian CSC spheroids from different patients exhibited varying, and personalized responses to chemotherapeutics. Xenografts were established from ovarian CSC spheroids, even with one single spheroid. Distinct responses to therapy were observed in distinct primary tumor xenografts similar to those observed in spheroids. Spheroids resistant to Cisplatin/ALDH inhibitor therapy had persistent, albeit lower ALDH expression and complete loss of CD133 expression while those resistant to cisplatin/JAK2 inhibitor therapy were enriched for ALDH+ cells [54].

Furthermore, in both published and unpublished results from our own laboratory we have proven, the diversity between responses of several human CRC cells to various anticancer agents, commonly used as a mainstay in early, as well as in advanced (metastatic) CRC treatment; the differences in expression level of various CRC-SC molecular markers, including ALDH1 isoforms, $\mathrm{ABC}$ transporters, plasma membrane receptors such as, Lgr5 and transcription factors such as, Nanog and SOX2, were correlated to the differences in chemotherapeutic effect of anticancer agents in human CRC cells. With multiple such experiments, we have indicated the increased cytotoxic effect of the drug Raltitrexed on HT-29 and HCT-116 CRC cells compared to the LS-180 CRC cell line- a result associating the different expression profiles of CSC markers, as a possible explanation of this phenomenon, since all three cells lines belong to the same cancer type, namely colorectal carcinoma. The same patterns account for the drug Irinotecan, while our experiments proved that the drug Capecitabine, follow the exact inverse pattern, exhibiting a much greater cytotoxic effect towards LS180 CRC cells [48].

Finally, we have also proven that CRC cells, rendered resistant to the drug 5-FU, change both their chemosensitivity towards the tested anticancer agents, as well as their levels and patterns of CRC-SC marker expression, with the above mentioned results pointing further to the potential of use of CSC and their marker in the personalization of treatment regimens in colorectal neoplasia, stratifying patients according to tumor-CRC-SC types, with the use of such biomarkers, both pre- and post- chemotherapy initiation $[48,57]$.

\section{Discussion}

In the prism of the evident need for new approaches in the field of cancer treatment and after due consideration of complications such as, anticancer treatment failure, the use of novel modalities for treatment adjustment and personalization, as well as patient grouping and stratification, according to the molecular characteristics and the biology of each, individual tumor, seems, indeed highly promising.

In this review, we systematically summarized, the most recent and updated experimental results, regarding the role of cancer stem cells, as well as other types of stem cells of the tumor microenvironment, in the context of treatment of colorectal neoplasia. After reviewing the current knowledge on the vast importance of this specific type of neoplasia worldwide, as well as its basic molecular and gross-pathological characteristics, we reviewed the mainstays in the CRC treatment nowadays including chemotherapy, surgical interventions and radiotherapy and thence, we analyze 
the main perplexities of current treatment, including concept of chemo- , radio- and multidrug resistance, which account for neoplastic lesion relapse, treatment failure and overall, decreased survival of CRC patients. This phenomena, clearly point the way to the applicability of personalized medicine in this field and in this context, we further analyze the up-to date research in the stem cell science field.

Citing results of research for the effects of mesenchymal stromal cells (MSCs) and thus, the association of tumor microenvironment aspects on CRC cells, we observe that, evidence pointing to a "tumor-promoting" role of MSCs is being opposed by recent evidence indicating an anti-tumor effect, which, however, appears to be specific for the very early stages of tumor development: stating this, administration of MSCs at this very early stage can have a tumor inhibiting effect by reducing inflammatory signaling and DNA damage in human colorectal cells. Furthermore, MSCs studied in the context of chemo- and radioresistance in CRC cells, seem to induce resistance to chemotherapeutics used in CRC, as seen in experiments using 5-Fluorouracil (5-FU), the treatment of choice for this cancer type. In addition, MSCs have been shown to be radio-resistant and so, could withstand such therapy and retain their tumor-conditioned phenotype, thus potentially contributing to disease relapse. However, genetically modified MSCs have been tested in the enzyme/prodrug therapy: this therapy can be selective against the neoplastic cells and the results of such studies concluded that, genetically modified MSCs, were successfully used in the treatment of colon cancer.

A vast amount of experimental research, moreover, indicated the role of cancer stem cells in novel treatment approaches for CRC, with a predictable extension of this concept in the treatment of many other types of neoplasia. Several molecules have been proposed as colorectal CSC markers, including CD133, CD44, CD24, CD166, Lgr-5, and aldehyde dehydrogenase 1 (ALDH1), CD166, epithelial cell adhesion molecule (EpCAM), CD29, CD24, CD26, Msi-1, Lgr-5 receptor, and Wnt activity/ $\beta$-catenin, among many others. Considering thus, the critical role of cancer stem cells in aspects like tumorigenicity, metastasis, chemo/ radioresistance and in many cases multidrug resistance in anticancer treatment, as well as the different molecular biology profiles of cancer stem cells in each individual tumor, research focused on such CSC associated molecules, serving as both, markers of recognition and separation, as well as targets for their selective treatment opting for decrease of tumor relapse, metastasis and anticancer treatment failure.

The treatment personalization potential of CSC markers in the clinic is promptly expanding, since it has been shown that CSCs and their associated markers can account for poor patient prognosis. Thus, CSC markers may not only be important for their identification or targeting but can also prove to be useful treatment personalization "tools"- an asset in proper selection of regimen of therapy and current status of treatment, serving as novel biomarkers in the battle against CRC.

According to the relevant, cited results of our research, we can observe the role of CRC stem cells and their molecular markers in conferring chemoresistance to several anticancer chemotherapeutics in certain types of CRC cells, with this phenomenon being whatsoever, variable, or even absent in other CRC cell types. The differences in expression level of various CRC-SC molecular markers, including ALDH1 isoforms, ABC transporters, plasma membrane receptors such as, Lgr-5 and transcription factors such as, Nanog and SOX2, were correlated to the variabilities in chemotherapeutic/cytotoxic effect of anticancer agents in human CRC cells, indicating their potential for future use as biomarkers in CRC treatment and thus therapeutic regimen individualization.

\section{Conclusions}

This review summarizes our knowledge of the effects and potential of stem cells and cancer stem cells in the field of personalized CRC treatment, serving as an indication of the importance of such research, in the development of novel, cancer therapeutic approaches, aiming for improving prognosis, survival and the patient's quality of life. Future perspectives, in the context of taking advantage of these reviewed research results, should opt to further verify the potential of cancer stem cells and their markers as well as stem cells in general, regarding CRC treatment personalization.

Author Contributions: A.P.; writing of the manuscript, Z. K; review and editing of the manuscript. 
Funding: This research received no external funding.

Acknowledgments The studies and experiments mentioned in this study were performed with the kind support provided by the Slovak Research and Development Agency under the contract APVV16-0178, Slovak Cancer Research Foundation; VEGA grant No 2/0124/17.

Conflicts of Interest: The authors declare no conflict of interest.

\section{References}

1. Thomassen, I.; van Gestel, Y.R.; Lemmens, V.E.; de Hingh, I.H. Incidence, prognosis, and treatment options for patients with synchronous peritoneal carcinomatosis and liver metastases from colorectal origin. Dis Colon Rectum 2013, 56, 1373-1380.

2. Colon, Rectal, and Anal Cancers. Availabe online: (accessed on

3. Garza-Trevino, E.N.; Said-Fernandez, S.L.; Martinez-Rodriguez, H.G. Understanding the colon cancer stem cells and perspectives on treatment. Cancer Cell Int 2015, 15, 015-0163.

4. Medical Biochemistry; Mosby Elsevier: 2009; Vol. 1.

5. De_Vita, V.T.; Lawrence, T.S.; Rosenberg, S.A. Cancer : principles \& practice of oncology; Wolters Kluwer Health : Lippincott Williams \& Wilkins: Philadelphia, 2015.

6. Gustavsson, B.; Carlsson, G.; Machover, D.; Petrelli, N.; Roth, A.; Schmoll, H.J.; Tveit, K.M.; Gibson, F. A review of the evolution of systemic chemotherapy in the management of colorectal cancer. Clin Colorectal Cancer 2015, 14, 1-10.

7. Fakih, M.G. Metastatic colorectal cancer: current state and future directions. J Clin Oncol 2015, 33, 18091824.

8. Aprile, G.; Lutrino, S.E.; Ferrari, L.; Casagrande, M.; Bonotto, M.; Ongaro, E.; Puglisi, F. Evidence-based appraisal of the upfront treatment for unresectable metastatic colorectal cancer patients. World $J$ Gastroenterol 2013, 19, 8474-8488.

9. Price, T.J.; Segelov, E.; Burge, M.; Haller, D.G.; Tebbutt, N.C.; Karapetis, C.S.; Punt, C.J.; Pavlakis, N.; Arnold, D.; Gibbs, P., et al. Current opinion on optimal systemic treatment for metastatic colorectal cancer: outcome of the ACTG/AGITG expert meeting ECCO 2013. Expert Rev Anticancer Ther 2014, 14, 1477-1493.

10. Mohelnikova-Duchonova, B.; Melichar, B.; Soucek, P. FOLFOX/FOLFIRI pharmacogenetics: the call for a personalized approach in colorectal cancer therapy. World J Gastroenterol 2014, 20, 10316-10330.

11. Xiang, B.; Snook, A.E.; Magee, M.S.; Waldman, S.A. Colorectal cancer immunotherapy. Discov Med 2013, 15, 301-308.

12. Koido, S.; Ohkusa, T.; Homma, S.; Namiki, Y.; Takakura, K.; Saito, K.; Ito, Z.; Kobayashi, H.; Kajihara, M.; Uchiyama, K., et al. Immunotherapy for colorectal cancer. World J Gastroenterol 2013, 19, 8531-8542.

13. Weiner, L.M.; Murray, J.C.; Shuptrine, C.W. Antibody-based immunotherapy of cancer. Cell 2012, 148, 1081-1084.

14. Pavlidis, E.T.; Pavlidis, T.E. Role of bevacizumab in colorectal cancer growth and its adverse effects: a review. World J Gastroenterol 2013, 19, 5051-5060.

15. Jiang, Z.; Li, C.; Li, F.; Wang, X. EGFR gene copy number as a prognostic marker in colorectal cancer patients treated with cetuximab or panitumumab: a systematic review and meta analysis. PLoS ONE 2013, 8, 18. 
16. F.S., L. Mechanisms of chemotherapeutic drug resistance in cancer therapy - A quick review. Taiwan J Obstet Gynecol September, 2009, pp 239-244.

17. Mimeault, M.; Hauke, R.; Batra, S.K. Recent advances on the molecular mechanisms involved in the drug resistance of cancer cells and novel targeting therapies. Clin Pharmacol Ther 2008, 83, 673-691.

18. Rajendra, P.N.; Muthusamy, G.; Shanmugam, M.; Ambudka, S.V. South Asian Medicinal Compounds as Modulators of Resistance to Chemotherapy and Radiotherapy. March 05, 2016, pp 32-36.

19. O'Malley, G.; Heijltjes, M.; Houston, A.M.; Rani, S.; Ritter, T.; Egan, L.J.; Ryan, A.E. Mesenchymal stromal cells (MSCs) and colorectal cancer: a troublesome twosome for the anti-tumour immune response? Oncotarget 2016, 7, 60752-60774, doi:10.18632/oncotarget.11354.

20. Niess, H.; von Einem, J.C.; Thomas, M.N.; Michl, M.; Angele, M.K.; Huss, R.; Günther, C.; Nelson, P.J.; Bruns, C.J.; Heinemann, V. Treatment of advanced gastrointestinal tumors with genetically modified autologous mesenchymal stromal cells (TREAT-ME1): study protocol of a phase I/II clinical trial. BMC Cancer 2015, 15, 237, doi:10.1186/s12885-015-1241-x.

21. Xin, H.; Sun, R.; Kanehira, M.; Takahata, T.; Itoh, J.; Mizuguchi, H.; Saijo, Y. Intratracheal delivery of CX3CL1-expressing mesenchymal stem cells to multiple lung tumors. Mol Med 2009, 15, 321-327.

22. Mueller, L.P.; Luetzkendorf, J.; Widder, M.; Nerger, K.; Caysa, H.; Mueller, T. TRAIL-transduced multipotent mesenchymal stromal cells (TRAIL-MSC) overcome TRAIL resistance in selected CRC cell lines in vitro and in vivo. Cancer gene therapy 2011, 18, 229-239.

23. Amara, I.; Touati, W.; Beaune, P.; de Waziers, I. Mesenchymal stem cells as cellular vehicles for prodrug gene therapy against tumors. Biochimie 2014, 105, 4-11.

24. Karjoo, Z.; Chen, X.; Hatefi, A. Progress and problems with the use of suicide genes for targeted cancer therapy. Adv Drug Deliv Rev 2016, 99, 113-128.

25. Nouri, F.S.; Wang, X.; Hatefi, A. Genetically engineered theranostic mesenchymal stem cells for the evaluation of the anticancer efficacy of enzyme/prodrug systems. J Control Release 2015, 200, 179-187.

26. Sorscher, E.J.; Hong, J.S.; Allan, P.W.; Waud, W.R.; Parker, W.B. In vivo antitumor activity of intratumoral fludarabine phosphate in refractory tumors expressing E. coli purine nucleoside phosphorylase. Cancer Chemother Pharmacol 2012, 70, 321-329.

27. Zielske, S.P.; Livant, D.L.; Lawrence, T.S. Radiation increases invasion of gene-modified mesenchymal stem cells into tumors. Int J Radiat Oncol Biol Phys 2009, 75, 843-853.

28. Colvin, H.S.; Nishida, N.; Koseki, J.; Konno, M.; Kawamoto, K.; Tsunekuni, K.; Doki, Y.; Mori, M.; Ishii, H. Cancer stem cells of the digestive system. Jpn J Clin Oncol 2014, 44, 1141-1149.

29. Insan, M.B.; Jaitak, V. New approaches to target cancer stem cells: current scenario. Mini Rev Med Chem 2014, 14, 20-34.

30. O'Brien, C.A.; Pollett, A.; Gallinger, S.; Dick, J.E. A human colon cancer cell capable of initiating tumour growth in immunodeficient mice. Nature 2007, 445, 106-110.

31. Ricci-Vitiani, L.; Lombardi, D.G.; Pilozzi, E.; Biffoni, M.; Todaro, M.; Peschle, C.; De Maria, R. Identification and expansion of human colon-cancer-initiating cells. Nature 2007, 445, 111-115.

32. Horst, D.; Kriegl, L.; Engel, J.; Kirchner, T.; Jung, A. Prognostic significance of the cancer stem cell markers CD133, CD44, and CD166 in colorectal cancer. Cancer Invest 2009, 27, 844-850.

33. Dalerba, P.; Dylla, S.J.; Park, I.K.; Liu, R.; Wang, X.; Cho, R.W.; Hoey, T.; Gurney, A.; Huang, E.H.; Simeone, D.M., et al. Phenotypic characterization of human colorectal cancer stem cells. Proc Natl Acad Sci U S A 2007, 104, 10158-10163. 
34. Tomita, H.; Tanaka, K.; Tanaka, T.; Hara, A. Aldehyde dehydrogenase 1A1 in stem cells and cancer. Oncotarget 2016, 7, 11018-11032.

35. Muraro, M.G.; Mele, V.; Daster, S.; Han, J.; Heberer, M.; Cesare Spagnoli, G.; Iezzi, G. CD133+, CD166+CD44+, and CD24+CD44+ phenotypes fail to reliably identify cell populations with cancer stem cell functional features in established human colorectal cancer cell lines. Stem Cells Transl Med 2012, 1, 592-603.

36. Kamachi, Y.; Kondoh, H. Sox proteins: regulators of cell fate specification and differentiation. Development 2013, 140, 4129-4144.

37. Zhou, Y.Y.; Zeng, F.Y. [Two vital transcriptional factors Oct-4 and Nanog to keep the pluripotency and self-renewal of stem cells and related regulation network]. Yi Chuan 2008, 30, 529-536.

38. Golubovskaya, V.M. FAK and Nanog cross talk with p53 in cancer stem cells. Anticancer Agents Med Chem 2013, 13, 576-580.

39. Ciurea, M.E.; Georgescu, A.M.; Purcaru, S.O.; Artene, S.A.; Emami, G.H.; Boldeanu, M.V.; Tache, D.E.; Dricu, A. Cancer stem cells: biological functions and therapeutically targeting. Int J Mol Sci 2014, 15, 8169-8185.

40. Gill, S.; Thomas, R.R.; Goldberg, R.M. Review article: colorectal cancer chemotherapy. Aliment Pharmacol Ther 2003, 18, 683-692.

41. Ong, C.W.; Kim, L.G.; Kong, H.H.; Low, L.Y.; Iacopetta, B.; Soong, R.; Salto-Tellez, M. CD133 expression predicts for non-response to chemotherapy in colorectal cancer. Mod Pathol 2010, 23, 450-457.

42. Kahi, C.J. Chromocolonoscopy for colorectal cancer screening: Dive into the Big Blue. J Interv Gastroenterol 2012, 2, 112-113.

43. Dallas, N.A.; Xia, L.; Fan, F.; Gray, M.J.; Gaur, P.; van Buren, G., 2nd; Samuel, S.; Kim, M.P.; Lim, S.J.; Ellis, L.M. Chemoresistant colorectal cancer cells, the cancer stem cell phenotype, and increased sensitivity to insulin-like growth factor-I receptor inhibition. Cancer Res 2009, 69, 1951-1957.

44. Todaro, M.; Alea, M.P.; Di Stefano, A.B.; Cammareri, P.; Vermeulen, L.; Iovino, F.; Tripodo, C.; Russo, A.; Gulotta, G.; Medema, J.P., et al. Colon cancer stem cells dictate tumor growth and resist cell death by production of interleukin-4. Cell Stem Cell 2007, 1, 389-402.

45. Cammareri, P.; Scopelliti, A.; Todaro, M.; Eterno, V.; Francescangeli, F.; Moyer, M.P.; Agrusa, A.; Dieli, F.; Zeuner, A.; Stassi, G. Aurora-a is essential for the tumorigenic capacity and chemoresistance of colorectal cancer stem cells. Cancer Res 2010, 70, 4655-4665.

46. Song, B.; Wang, Y.; Xi, Y.; Kudo, K.; Bruheim, S.; Botchkina, G.I.; Gavin, E.; Wan, Y.; Formentini, A.; Kornmann, M., et al. Mechanism of chemoresistance mediated by miR-140 in human osteosarcoma and colon cancer cells. Oncogene 2009, 28, 4065-4074.

47. Ganoth, A.; Merimi, K.C.; Peer, D. Overcoming multidrug resistance with nanomedicines. Expert Opin Drug Deliv 2015, 12, 223-238.

48. Kozovska, Z.; Patsalias, A.; Bajzik, V.; Durinikova, E.; Demkova, L.; Jargasova, S.; Smolkova, B.; Plava, J.; Kucerova, L.; Matuskova, M. ALDH1A inhibition sensitizes colon cancer cells to chemotherapy. BMC Cancer 2018, 18, 656, doi:10.1186/s12885-018-4572-6.

49. Tu, L.C.; Foltz, G.; Lin, E.; Hood, L.; Tian, Q. Targeting stem cells-clinical implications for cancer therapy. Curr Stem Cell Res Ther 2009, 4, 147-153.

50. Ailles, L.E.; Weissman, I.L. Cancer stem cells in solid tumors. Curr Opin Biotechnol 2007, 18, 460-466.

51. Kawasaki, B.T.; Farrar, W.L. Cancer stem cells, CD200 and immunoevasion. Trends Immunol 2008, 29, 464-468. 
52. Saigusa, S.; Tanaka, K.; Toiyama, Y.; Yokoe, T.; Okugawa, Y.; Koike, Y.; Fujikawa, H.; Inoue, Y.; Miki, C.; Kusunoki, M. Clinical significance of CD133 and hypoxia inducible factor-1alpha gene expression in rectal cancer after preoperative chemoradiotherapy. Clin Oncol 2011, 23, 323-332.

53. Kreso, A.; Dick, J.E. Evolution of the cancer stem cell model. Cell Stem Cell 2014, 14, 275-291.

54. Raghavan, S.; Mehta, P.; Ward, M.R.; Bregenzer, M.E.; Fleck, E.M.A.; Tan, L.; McLean, K.; Buckanovich, R.J.; Mehta, G. Personalized Medicine-Based Approach to Model Patterns of Chemoresistance and Tumor Recurrence Using Ovarian Cancer Stem Cell Spheroids. Clin Cancer Res 2017, 23, 6934-6945.

55. Krause, M.; Dubrovska, A.; Linge, A.; Baumann, M. Cancer stem cells: Radioresistance, prediction of radiotherapy outcome and specific targets for combined treatments. Adv Drug Deliv Rev 2017, 109, 6373.

56. Glinsky, G.V. "Stemness" genomics law governs clinical behavior of human cancer: implications for decision making in disease management. J Clin Oncol 2008, 26, 2846-2853.

57. Durinikova, E.; Kozovska, Z.; Poturnajova, M.; Plava, J.; Cierna, Z.; Babelova, A.; Bohovic, R.; Schmidtova, S.; Tomas, M.; Kucerova, L., et al. ALDH1A3 upregulation and spontaneous metastasis formation is associated with acquired chemoresistance in colorectal cancer cells. BMC Cancer 2018, 18, 018-4758. 